\title{
Interactive comment on "The significance of vertical moisture diffusion on drifting Snow sublimation near snow surface" by Ning Huang and Guanglei Shi
}

\section{Ning Huang and Guanglei Shi}

shigl14@|zu.edu.cn

Received and published: 4 September 2017

Dear Dr. Ally Toure, Thank you for your comments concerning our manuscript entitled 'The significance of vertical moisture diffusion on drifting snow sublimation near snow surface'. We deeply appreciate the time and effort you have spent in reviewing our manuscript. We will carefully consider each comment, and make cautious revision accordingly. Below are our point-to-point responses.

Item 1: The objectives of the paper are clear. However the connections between equations are not always clear to the reader. Response: Thanks for the comment. Following your suggestion, we will add more descriptions on the connections between equations 
in the revised manuscript.

Item 2: Also, I understand the challenge of finding ground-based observations to valide simulation of the sublimation. But the limited number of observations used here weakens the conclusion made by the authors. In other words the lack of sufficient validation data makes it impossible to say for certain if this model constitute an improvement over the previous models. Response: Thanks. Just as you said, ground-based observations sublimation observations are few. However, we still found some experimental results that could be used to validate our model. For example, we compared our simulated snow sublimation rate with that of Schmidt's observational results (see Fig. 3). We also compared the simulated snow mass concentration with that of Pomeroy and Male (1992) to indirectly validate our model (see Fig. 2).

Item 3: Line 7 : instead of "drifting snow sublimation" the authors could clear state "sublimation of blowing snow" or "sublimation of transported snow particles". Response: Thanks for your useful suggestion. We will replace the phrase drifting snow sublimation with sublimation of blowing snow in the revised manuscript.

Item 4: Line 10: by "snow sublimation near surface " Do the authors mean the sublimation during the saltaion phase or the turbulent suspension phase of the blowing snow or both? Response: In this manuscript, "snow sublimation near surface" includes the sublimation of both saltation particles and turbulent suspension particles in the region which is close to the snow bed.

Item 5: Line $10-11$ : I would say that the statement is not exactly correct. There are a few models that take this sublimation of blowing snow into account (see for example Liston and Sturm, 1998, Essery et al., 1999). Response: Thanks for the comment. You are right. Although in most of models snow sublimation near surface was ignored, some models did consider the sublimation of near bed. But in these models the value of sublimation near surface is only a rough estimate by some empirical formula based on assumptions. We will comment on previous work with more precise sentences in

Printer-friendly version

Discussion paper 
the revised manuscript.

Item 6: Line 15: the sentence is not clear. Response: Thanks for your suggestion. We will modify the sentence so that its meaning will be clearly expressed in the revised manuscript.

Item 7: Line 17: How small? Response: From Fig. 12, we can see that the mass of snow sublimation near surface accounts for even more than half of the total when the friction wind velocity is less than about $0.55 \mathrm{~m} / \mathrm{s}$.

Item 8: Line 20: this sentence need rewording Response: Following your suggestion, we will reword this sentence in the revised manuscript.

Item 9: Line 42: need to cite references : "Many researchers. ...(references)" Response: Thanks for your carefully reviewing of the manuscript. We will add some relevant references in the revised manuscript.

Item 10: Line 43: is "violently" the appropriate scientific word to use here? Response: Thanks. As your suggestion, the sentences in line 43-45 will be modified in the revised manuscript as: Many researchers believed that the snow particles sublimation near surface would be great at the early stage of drifting snow process. However, the high concentration of snow particles near surface would result in a rapid air temperature decrease and humidity increase. Therefore the humidity would reach saturation quickly near surface, and the sublimation would stop at the saturated layer of humidity.

Item 11: Line 48: I would state this: "However, some researchers (references) found that humidity near surface not to reach saturation in the drifting snow in the field, ....." Response: Thanks. Following your suggestion, we will add some references in the revised manuscript.

Printer-friendly version

Item 12: Line 68: "But this model can not describe snow particles suspending in upper air." The sentence is awkward and need rewording. Response: Thanks. Following your suggestion, we will modify this sentence in the revised manuscript. 
Item 13: Line 85: Authors should explain why use Flows equation instead of Blowing snow equation in for example Liston et Sturm, 1998. Response: In order to accurately calculate the sublimation mass of snow particles, we need to know detailed information of each snow particles in the air (including particle size, relative velocity of particles to the wind speed, etc.). These data can't be directly obtained from the blowing snow equation. But they can be calculated by the flow equation and the snow particle motion equation.

Item 14: Line 94: judging criterion?? Do the authors mean "Threshold". Response: "judging criterion" is the criterion for judging whether a particle is a saltation particle or a suspended particle.

Item 15: line 97: the authors should show the connection between this equation and the previous ones (eqs 1and 2). Response: Thanks. This equation is used to calculate the final sedimentation velocity, which is a parameter in Equation 2. We will explain it in the revised manuscript.

Item 16: Line 121: Please add reference. Response: Thanks. As your suggestion, we will add some references in the revised manuscript.

Item 17: Line 171: Which particles, please explain. Response: Thanks. The snow particle size distribution is that we used in the blowing snow model. We will explain it in the revised manuscript.

Item 18: Line 185: This conclusion is based on only 4 observations of field is a little bit of a stretch. For example, there is no observations on the figures $2 \mathrm{a}, \mathrm{b}, \mathrm{c}$. Response: The reviewer is right. Because of the limited observational conditions, only a few observations are generally available. And we can only validate our model with such a limited number of observations.

Item 19: Line 189: What are those environmental conditions? Can the authors explain why the difference What is the difference between the authors approach and that 0 
schmidt? Response: The conditions used in our simulations are the same as those reported by Schmidt. And we will add the environmental conditions in the revised manuscript. Actually the conditions in the field are much complex and changing fast. Therefore it is almost impossible that the results of numerical simulation and field observation results are exactly the same. Nevertheless, it can be seen from Fig. 3 that the two results are relatively consistent, so we think that our model is reliable.

Item 20: Line 195: What are the difference between the 4 models apart from that the all neglect sublimation? Response: The differences among these four models mainly includes structure of the models, numerical methods, meteorological field treatment and the parameterization schemes although they are based on common physical concepts. Detailed information can be found in the paper by Xiao et al. (2000).

Item 21: Lines 200: - 205: this section is not clear and need to be reworded Response: Thanks. As your suggestion, we will reword this sentence.

Item 22: Line 206: "suspended particles versus various friction velocities" IT should be "for various ..." instead of "versus, ..." Response: Thanks. As your suggestion, we will modify this word.

Item 23: Line 209: "reach stead" what does that mean? do you mean "plateau" = constant value? Response: Yes, it means that the values of parameters, such as mass of saltation particles and suspension particles will not change with time.

Item 24: Line 214-216: This sentence is incomprehensible Line 219: Need rewording Line 221: Need rewording too Line 227: "reach steady" is not an appropriate phrase to use in my view. Line 230: need rewording Line 246: The sencence need rewording Response: As your suggestion, we will reword these sentences. Actually, we asked a native English speaker, who is an English teacher in my university will revise the English of this manuscript.

Item 25: Line 270: I don't think this statement is true. Many models do take the sub-

Printer-friendly version

Discussion paper 
limation into account Response: Thanks for the comment. You are right. Although in most of models snow sublimation near surface was ignored, some models did consider the sublimation of near bed. But in these models the value of sublimation near surface is only a rough estimate by some empirical formula based on assumptions. We will Comment on previous work with more precise sentences in the revised manuscript.

Item 26: Line 275: Pomeroy et Male (1992)??? Vionnet et al. (year???) Response: Thanks for your comment. We will add the year in the revised manuscript.

Item 27: Line 284: Could the authors cite the study that neglected the saltation? Response: Thanks. As your suggestion, we will add the references in the revised manuscript.

Item 28: Figure 13: The smaller figure is not readable Response: Thanks for your comment. We will modify this figure.

Item 29: Line 307: "Bellowing snow" what does that need? Response: Thanks for your comment. We will modify this word.

Once again, thank you very much for your comments and suggestions. Best regards Ning Huang and Guanglei Shi

Interactive comment on The Cryosphere Discuss., https://doi.org/10.5194/tc-2017-116, 2017. 